\title{
The Road Traffic Meteorological Monitoring and Forecasting System
}

\section{Based on GIS}

\author{
Yanlong $\mathrm{Chu}^{1}$ \\ ${ }^{1}$ Police Administration Department, Liaoning Police Academy, Dalian Liaoning China, 116036
}

KEYWORDS: Road Traffic, Meteorological Monitoring and Forecasting, GIS

\begin{abstract}
Traffic safety is the top priority of road traffic, weather and road traffic safety threat is huge. Rain, snow and other weather conditions affect people's lives become, the impact of national economic construction and development obstacles. Therefore, establishing a road traffic meteorological GIS system is a top priority.
\end{abstract}

\section{Introduction}

Since the reform and opening up, China's economic development speed very quickly, and will maintain rapid economic development of the situation in the next period of time, and the formation of a larger transportation pressure, which will require the development of road transport to keep up with rapid economic development the need for highway traffic is very susceptible to weather conditions. Due to lack of good means of access to the latest weather information and accurate, detailed weather road transport system and query system, the country from all aspects of the human, material and financial resources suffered heavy losses, but also to make the masses of my country Highway traffic safety trust has been at a low level. Traffic safety is the top priority of road traffic, weather and road traffic safety threat is huge. Rain, snow and other weather conditions affect people's lives become, the impact of national economic construction and development obstacles.

The establishment of a road traffic meteorological GIS system a top priority. This system can analyze and query the meteorological conditions of the road, and can check weather conditions each time period in the past and you can keep abreast of the weather conditions and even sections of the target area, and make appropriate countermeasures in advance. You can minimize road accidents. This study is a road traffic and weather combine the GIS platform requires transport sector and meteorological departments to cooperate in providing relevant information. Query system fully open to the public so that the public understand highway traffic weather conditions.

\section{The Impact of Meteorological Conditions on Road Transport}

Effects of meteorological conditions on traffic performance are in many ways. From the impact mechanism side, the main aspects of traffic safety aspects of the impact of a vehicle condition, road conditions, traffic conditions, driving environment and the driver's judgment and ability to reflect, in different weather conditions and the impact on traffic mechanisms are different . Major disasters and the impact are:

Mainly through the fog reduce visibility and cause accidents. Cause malignant traffic accidents in most parts of China weather phenomenon, the greatest impact of fog. Fog especially $<5 \mathrm{~m}$ ultra low visibility caused by fog disaster is an important cause of major accidents, often caused by 
continuous rear-end the number of vehicles even dozens of cars, the local presence or sudden "fog" phenomenon It is caused by "Knocking" culprit. Fog vehicles often cause significant losses and casualties, leading to highway speed limit or shut down travel time delays, resulting in huge economic losses. ${ }^{\circ}$

But also affect rainfall Highway Traffic Safety The most common meteorological elements, it makes the road adhesion coefficient is reduced, resulting in increased vehicle braking distance, prone vehicle skidding out of control and thus endanger traffic safety. While rain reduced visibility, the driver line of sight mode is lake unclear, leading to driver error. In addition, after the rain, wet pavement if water or mixed, uneven road surface friction coefficient, the vehicle brake deteriorates, causing a traffic accident.

Snowfall and rainfall, like the flying snow reduced visibility, and once the pavement or compacted snow melting in the sunlight during the day, cool at night pavement freeze, causing significant reduction in road surface friction coefficient, and seriously affect the operation of the vehicle system dynamic performance, so out of control, vehicle idling occurs, slipping or sliding, thus endangering traffic safety.

The vehicle running resistance, energy consumption and resistance to lateral tipping and anti-slip performance has a significant impact, especially on the high side wind box, box car with double the impact is even worse. Winds cause dust storms, blowing sand, blowing snow, dust and other weather, road visibility. In recent years, the number of dust storms increases, the intensity increased, the scope expanded, with the increase in traffic, will become the new road traffic hazards.

When the road surface frost, the road surface friction coefficient close to the snow surface, the physical properties of freezing rain with snow surface ice, as the cause of traffic accidents with snow.

Hot weather makes the vehicle failure rate was significantly increased and it may cause the car engine mixed. Spontaneous, blew. At the same time affected heat, friction and car exhaust, etc., Highway temperature is much higher than the temperature, sometimes as high as sixty to seventy degrees, so the heated car tires, inner tire pressure increased, long-distance high-speed, can easily cause / Explosion 0 tire. High temperature directly affects the physical, psychological and mental state of the driver, non-air-conditioned car more fatigue, inability to concentrate or even heat stroke.

\section{Necessity of GIS-Based Road Traffic Meteorological Monitoring and Forecasting System}

Property damage and casualties shocking Traffic Weather disasters have become the focus of the society, to ensure traffic safety, efficient, rapid and urgent need of comprehensive, multi-functional, intelligent weather protection. According to meteorological services carry traffic characteristics, to meet the full range, multi-functional, intelligent meteorological support requirements, development of the system should have the following functional attributes: (1) based on GIS technology, the provincial, city and county administrative regions plane data as a background layer, highways, national highways, road line data as live weather monitoring, forecasting and early warning service Enquiry important layer display; (2) real-time display and statistical basis of meteorological information and weather monitoring information highway of inquiry; (3) under the early warning indicator system, when severe weather occurs automatic alarm system; (4) the use of GIS technology and disaster prevention measures library that implements the forecasting and warning products once editing, auto-complete different channels and different customer service products intelligent distribution; (5) the use of management authority, to achieve different clients have different browsing service products; (6) enhance the capacity expansion by adding basic 
information to realize service object, add any service products and road weather monitoring stations and definitions.

\section{System Function}

Meteorological monitoring system includes a high-speed live-acquisition, traffic weather forecasting and warning services and products produced products receiving display of three parts. Road weather monitoring live transmission by radio to the Traffic Weather Operations Centers, traffic weather command center responsible for traffic weather monitoring live monitoring and forecasting and warning services, each Highway Administration through VPN leased line for high-speed monitoring live synchronize information and forecasting and early warning products receiving display.

The platform for forecasting services to staff and Highway Administration, with a road weather monitoring live-acquisition, fault monitoring, historical data analysis queries, can be a single station multi-element, multi-station single elements and the like in any way statistical inquiry, output, including charts, graphic, curves; achieve traffic, weather monitoring both live sync shared, manually set the warning threshold, the data refresh rate $5 \mathrm{~min}$, when monitoring a live pre exceeds a preset value, through various graphics and sound alarm alert personnel.

Overall system using VS2005 + GIS + Oracle11g platform developed using B / S architecture, dynamic effects in some places using Java script scripts to automatically generate key technology of interactive visualization and production forecasts contour map by drawing circle area way, a combination of internal system of early warning indicators and forecasting and early warning defense libraries for different clients within the circle drawing region to automatically distribute the appropriate product forecasting and warning services.

The system uses a modular structure, based on advanced, secure, scalable and systematic development and design principles. The system uses a layered architecture design, divided into: data layer, business logic layer, presentation layer. Data layers including automatic station data, rain intelligence text, disaster packets, road closure information, video information, regular forecasts, elements of forecasting, numerical prediction, base map data, thematic map data, warning indicator system libraries and defenses libraries. The main function of business logic is: analysis data storage layer data, regular forecasts and special forecasting tools management background.

Live display module. Live show is divided into four sub-modules: live monitoring, disaster live, road closure information, video information module. Live monitoring including radar, satellites, automatic weather stations and road weather monitoring information. Road weather monitoring routine feature update frequency, road conditions every minute update frequency of 2 minutes; with a variety of display graphics, curves, forms, etc., can achieve a variety of hierarchical and superimposed live information display.

Forecast production module. Forecast production is the core function of the system. In addition to the callable MICAPS V3.0 and VIPS forecasting system also incorporates a low visibility, icy road, pavement temperature, fine forecasting, transportation and other special short-impending prediction and forecasting tools based distribution point, line, surface intelligent forecast technology. Not only can be achieved in conventional, long and short-term forecast calls product and fast editing, more central, the system uses the screen positioning technology, once for all sites in selected regions of Meteorological Elements batch editing, combined.

Warning Production process: automatic warning - Correct manually - expert consultations - the issue of leadership - warning issued. The system automatically generates warning information under the early warning indicators within the system, and the way pictures and sound dual alarm prompts 
to use staff, through artificial revised warning level and warning ranges for major disasters process, you can start the Meteorological SMS publishing platform and Traffic consultation with sector experts or start police, traffic police, visual meteorological tripartite consultation when expert consultation feedback comments, feedback system itself will pop-up interface, early warning technology with the production and distribution of forecast production module.

Expert feedback and demand analysis module. Storage and analysis of traffic experts, users, public feedback and Traffic Weather Service site visits. Professional meteorological service website has left feedback interfaces for user feedback and suggestions at any time. Through regular feedback on a variety of information analysis, meteorological services help understand the actual needs of users, improve service satisfaction.

System Administration module system based on a modular architecture, adding new clients and road weather monitoring stations geographic information technology, forecasting tools and a variety of special leave repository interfaces, such as: management services for objects and each serve key service points. Defensive measures library. , Facilitate the revision and expansion of key technologies, the system remains advanced. This function reflects the strong expansion of the system.

\section{Conclusion}

Business operations through the system, and continuously improve and perfect the system, in particular to strengthen the impact of response measures and severe weather forecasting technology research and highway traffic, to make the system more powerful, more accurate service measures for public travel and highway departments to provide better services, improve the social and economic benefits.

\section{Acknowledgements}

Proj: General Research Project of 2015 Liaoning Province, Department of Education Science, Road Traffic Meteorological Monitoring and Warning System based on GIS.

Project NO.: L2015246.

\section{Reference:}

[1] Panya Ying, Chen Wu. Road traffic accident caused by weather conditions analysis [J]. Meteorological Science and Technology, 2006,34 (6): 778-782.

[2] Huangyu Ren, Shen Ying, Huang Yusheng, et al. Effects of urbanization on radiation fog in Xishuangbanna [J]. Plateau Meteorology, 2001,20 (2): 186-190.

[3] Like Wu Ping. Shanghai-Nanjing Expressway Weather Decision Support System [J] Modern Transportation Technology, 2006, (5): 91-94.

[4] Yang Xueying. Establishment of road weather warning mechanism, reduce traffic LCCA [J]. Foreign Highway, 2005,25 (6): 164-166.

[5] Von China school, Yuancheng Song, Bian Guanghui, and the like. Shanghai-Nanjing Expressway, Wuxi SPRING fog and a number of real-time monitoring features [J]. Meteorological Sciences, 2003,23 (4): 435-445.

[6] Tian Xiaoyi, Wu Jianjun, Yanming Liang, et al. Some New Progress in low visibility fog highway monitoring forecast [J]. Meteorological Sciences, 2009,29 (3): 415-420. 